\title{
Apparent diffusion coefficient as an indicator of spinal cord compression due to anterior atlanto-axial subluxation in rheumatoid arthritis patients
}

\author{
Małgorzata Mańczak ${ }^{1}$ (1) $\cdot$ Grzegorz Pracoń2 $^{2}$ Iwona Sudoł-Szopińska ${ }^{2} \cdot$ Robert Gasik $^{3}$
}

Received: 2 August 2018 / Revised: 24 June 2019 / Accepted: 30 June 2019 / Published online: 12 July 2019

(c) The Author(s) 2019

\begin{abstract}
Purpose The aim of this retrospective study was to evaluate the apparent diffusion coefficient (ADC) as a potential parameter of spinal cord damage in cervical spine instability at the atlanto-axial level in rheumatoid arthritis (RA) patients.

Methods One hundred and six RA patients were included in the study. MRI examinations were performed with 1.5T scanner. The ADC was measured at six locations in the cervical spinal cord at the height of the first six cervical vertebrae (from C-1 to C-6). The ADC values were assessed in 2 groups: with and without anterior atlanto-axial subluxation (AAS) diagnosed on plain radiographs. Correlations between ADC values and radiographic measurements and RA activity indicators were evaluated.

Results The $\mathrm{ADC}$ values at $\mathrm{C} 1$ level $\left(\mathrm{ADC}_{1}\right)$ was higher in the group with anterior AAS than in the group without AAS $(p<0.001)$. Statistically significant moderate positive correlation between $\mathrm{ADC}_{1}$ and anterior atlanto-axial diameter interval AADI (rho $=0.58 ; p<0.008$ ) was found as well as statistically significant weak negative correlation between $\mathrm{ADC}_{1}$ and posterior atlanto-axial diameter interval PADI ( $r$ ho $=-0.34 ; p<0.008)$.

Conclusions The conducted study demonstrates the applicability of the ADC maps in the identification of spinal cord compression due to anterior AAS in RA patients. The results encourage the practical use of the ADC as an additional parameter in the qualification for surgical treatment.
\end{abstract}

\section{Graphic abstract}

These slides can be retrieved under Electronic Supplementary Material.
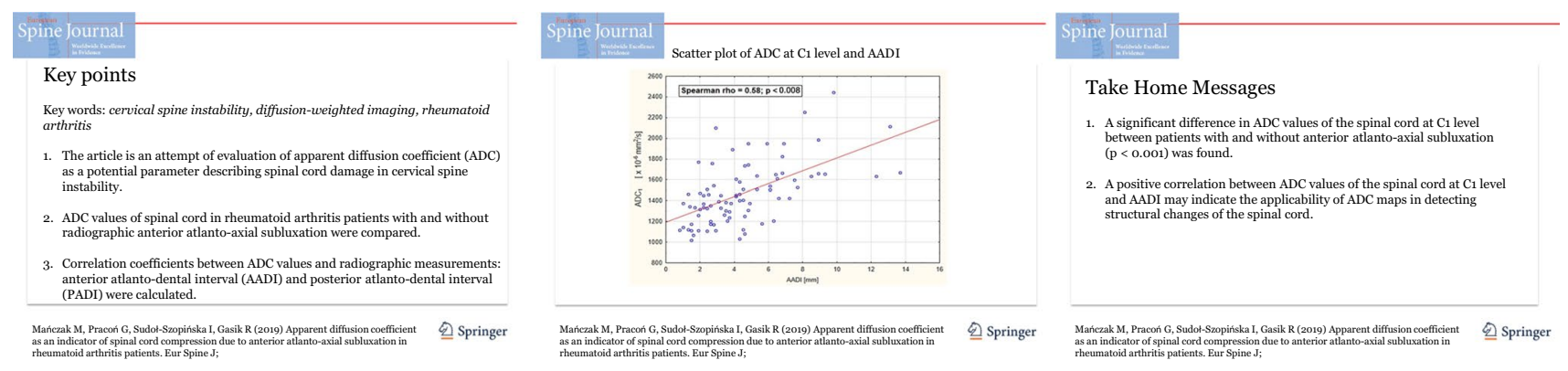

Keywords Cervical spine instability $\cdot$ Magnetic resonance $\cdot$ Diffusion-weighted imaging $\cdot$ Rheumatoid arthritis

Electronic supplementary material The online version of this article (https://doi.org/10.1007/s00586-019-06058-2) contains supplementary material, which is available to authorized users.

Małgorzata Mańczak

m.manczak@op.pl

Extended author information available on the last page of the article

\section{Introduction}

Rheumatoid arthritis (RA) is a chronic systemic inflammatory disease affecting $1-2 \%$ of the world's population [1-3]. The cervical spine is the third most commonly affected 
region, following hands and feet. Pathology in the cervical spine is observed in $44-86 \%$ of patients with RA [4, 5]. The most debilitating abnormality is the excessive movement at the junction between the atlas $(\mathrm{C} 1)$ and axis $(\mathrm{C} 2)$ called an atlanto-axial subluxation (AAS), which occurs in about $65 \%$ of patients and includes the anterior, posterior, lateral instability and the basilar invagination [6].

The AAS in the course of RA is the result of the activity of inflamed synovium, the pannus, which leads to ligamentous damage (the transverse ligament and alar ligaments) and also to bony erosions. The diagnosis of the anterior AAS is based mainly on two imaging methods: plain radiography and magnetic resonance imaging (MRI). The initial evaluation of the atlanto-axial joint in relation to the anterior AAS is based on the functional lateral radiographs in neutral, flexion and extension positions. The assessment consists of measuring two distances: the anterior atlantodental interval (AADI) and the posterior atlantodental interval (PADI).

The lateral radiographs of the cervical spine in flexion are of the utmost importance for the diagnosis of the anterior AAS. Anterior head tilt leads to the displacement of the dens which on lateral radiographs is seen as an increase in AADI and a decrease in PADI. The normal AADI in a healthy adult is considered to be less than $3 \mathrm{~mm}$. A reduction of PADI to less than $14 \mathrm{~mm}$ indicates a critical reduction of the space around the spinal cord.

In the case of any radiographic pathologies, MRI of the cervical spine is recommended [7]. It is considered that hyperintensity on T2-weighted images (and other water-sensitive sequences) and hypointensity on T1-weighted images within the spinal cord at the level of compression are highly suggestive of its severe damage. Both clinical practice and numerous studies indicate that there is no clear correlation between the disease severity and cervical spine pathology on imaging. More than half of RA patients with instability on radiographs do not present any neurological ailments or symptoms $[8,9]$. On the other hand, many patients even with clinically significant stenosis of the spinal canal do not show any pathology on imaging studies, including no T2-weighted hyperintensity or T1-weighted hypointensity on MRI, especially at the atlanto-axial level $[10,11]$. This causes great difficulties in planning the treatment and deciding on a surgical stabilization of the spine.

New techniques in MRI are expected to allow the evaluation of the spinal cord pathology before irreversible changes appear. One of them is the diffusion-weighted imaging (DWI), which shows the movement of water molecules (diffusion) in the tissue. In this technique, two images are generated: isotropic diffusion map often called DWI image and an apparent diffusion coefficient (ADC) map describing a magnitude of diffusion in the visualized tissues.

The aim of the study was to evaluate the ADC as a potential parameter of spinal cord damage in cervical spine instability at the atlanto-axial level in RA patients by comparing ADC values in patients with and without radiographic anterior AAS, and to correlate ADC values with radiographic measurements and some RA clinical outcomes.

\section{Method}

\section{Patients}

One hundred and ten consecutive RA patients who underwent DWI MRI of the cervical spine were recruited to this retrospective study. Inclusion criteria included the age of at least 18, clinical diagnosis of RA and DWI MRI of the cervical spine performed in the diagnostic process. Exclusion criterion was previous cervical spine surgery. Movement artifacts found on DWI images excluded four patients from the analysis. Finally, 106 patients (94 women and 12 men; median age 60 years (IQR: 51-68), median RA duration 13.5 years) were included to the study. The protocol of the study was approved by local bioethical committee.

\section{MRI}

All MRI examinations were performed with SIEMENS Avanto 1.5T. The protocol included conventional sagittal and axial T1- and T2-weighted sequences and sagittal DWI sequences. DWI images were obtained with the following parameters: $\mathrm{TR}=3300 \mathrm{~ms}, \mathrm{TE}=82 \mathrm{~ms}, \mathrm{FOV}=400 \mathrm{~mm}$, voxel size $=2.6 \times 2.1 \times 3.0 \mathrm{~mm} ; 17$ slices with $3 \mathrm{~mm}$ thickness. DWI was performed using single-shot echo-planar imaging (EPI) with three $b$-values: 50, 400 and $800 \mathrm{~s} / \mathrm{mm}^{2}$.

\section{Image analysis}

Neither T2 hyperintensity nor T1 hypointensity within the spinal cord in atlanto-axial region was observed in 106 evaluated cases. The ADC was measured at six locations of the cervical spinal cord in each patient, at the height of six following cervical vertebrae: from C-1 to C-6. Each measurement of the ADC, using the region of interest (ROI), which was a circle with a homogeneous signal and an area of about $0.26 \mathrm{~cm}^{2}$, was taken three times, and the average value was calculated. The AADI and PADI values were measured on lateral radiographs in flexion. The AADI was defined as the distance between the posterior surface of the anterior arch of the atlas and the anterior surface of the dens of the axis. The PADI was defined as the distance between posterior surface of the dens and the anterior surface of the posterior arch of the atlas.

The study population was divided into two groups based on the AADI measurement: group I-patients with the anterior instability in the atlanto-axial joint 
(AADI $>3 \mathrm{~mm}$ ) and group II-patients without the anterior instability in the atlanto-axial joint (AADI $\leq 3 \mathrm{~mm}$ ).

The following patients' data were collected from the hospital patient base: sex, age, disease duration, erythrocyte sedimentation rate (ESR), C-reactive protein level (CRP), disease activity score (DAS-28) and neurological examination results.

\section{Statistical analysis}

As a preparation for the study, an estimate of the minimum sample size was made. We assumed that it would be satisfactory to show a difference in the ADC values of about $200 \times 10^{-6} \mathrm{~mm}^{2} / \mathrm{s}$ between the evaluated groups. Assuming a test strength of 0.90 and a statistical significance level of 0.05 , the estimated minimum number of each group was about 45 cases. Thus, an analysis of images of 100 consecutive patients who met the inclusion criteria was planned.

The statistical analysis was carried out with Statistica10. The normality of the distribution of continuous variables was verified using the Shapiro-Wilk test. The distribution of all continuous variables analyzed in the study differed significantly from normal distribution, and therefore, median and interquartile range (IQR) were used. Mann-Whitney U test was used to compare continuous variables. Bonferroni correction was applied due to multiple comparisons, and the level of statistical significance was set at $p<0.008(0.05 / 6 \approx 0.008)$. Correlations between variables were investigated using Spearman's rank correlation analysis. Correlation coefficients at $p<0.008$ (in accordance with Bonferroni correction) were considered statistically significant.

An analysis of the ROC (receiver operating characteristic) curve has been carried out, determining the optimal cutoff point for ADC value as a criterion of instability. Sensitivity, specificity, positive predictive value (PPV) and negative predictive value (NPV) of the created diagnostic test were calculated.

\section{Results}

\section{Comparison of ADC between RA patients with and without instability}

The median and interquartile range of the ADC values for the six levels of cervical spine in two analyzed groups are presented in Table 1. There is a significant difference in the $\mathrm{ADC}$ at $\mathrm{C} 1$ level $(p<0.001)$ : The $\mathrm{ADC}$ is higher in the group with the anterior AAS.
Table 1 The ADC values in spinal cord at six consecutive vertebrae levels ( $\mathrm{C} 1$ to $\mathrm{C} 6)$ in patients with the anterior atlanto-axial subluxation (group I) and without subluxation (group II)

\begin{tabular}{llll}
\hline Parameter & $\begin{array}{l}\text { Group I } \\
n=53\end{array}$ & $\begin{array}{l}\text { Group II } \\
n=32\end{array}$ & $p$ \\
& Median (IQR) & Median (IQR) & \\
\hline $\mathrm{ADC}_{1}$ & $1507(1374-1658)$ & $1330(1133-1461.5)$ & 0.0003 \\
$\mathrm{ADC}_{2}$ & $1187(1125-1314)$ & $1155.5(1043-1219)$ & 0.0395 \\
$\mathrm{ADC}_{3}$ & $1039(965-1117)$ & $1035(960-1111.5)$ & 0.9748 \\
$\mathrm{ADC}_{4}$ & $1003(933-1073)$ & $973(920.5-1054.5)$ & 0.3452 \\
$\mathrm{ADC}_{5}$ & $986(929.5-1044)$ & $966.5(926.5-1082.5)$ & 0.9892 \\
$\mathrm{ADC}_{6}$ & $983(934-1063)$ & $1003.5(928-1102.5)$ & 0.4348 \\
\hline
\end{tabular}

\section{Correlation between $A D C$ values, AADI and PADI as well as clinical parameters}

Statistically significant moderate positive correlation between the $\mathrm{ADC}_{1}$ and the AADI (rho = 0.58) was found as well as statistically significant weak negative correlation between the $\mathrm{ADC}_{1}$ and the PADI (rho = -0.34) (Fig. 1a, b). No other correlations between the ADC values (at $\mathrm{C} 2-\mathrm{C} 6$ levels) and the AADI or the PADI were found. No statistically significant correlation was found between ADC values at six levels of cervical spine and patient age, disease duration, ESR, CRP and DAS-28.

There were also no differences in ADC values in patients with and without neurological symptoms: pain, pyramidal symptoms, sensory symptoms (all $p>0.05$ ).

\section{ROC analysis}

ROC analysis for $\mathrm{ADC}_{1}$ as a predictor of anterior atlantoaxial subluxation was performed (Fig. 2). The area under curve (AUC) which is a measure of discriminative capacity of a given parameter is $0.730(\mathrm{SE}=0.057)$.

Sensitivity, specificity, PPV and NPV for several selected cutoffs of ADC1 are given in Table 2. In the first line of the table, the cutoff value calculated using the Youden index is given. In the following lines, there are cutoff points for which the specificity and PPV are the highest.

\section{Discussion}

There are many studies evaluating cervical spinal cord pathology, especially in degenerative cervical myelopathy by means of diffusion-weighted and diffusion tensor imaging (DTI). In our work, we explore the potential role of ADC maps in identification of spinal cord compression in RA patients. The results of our study show that ADC values at $\mathrm{C} 1$ level are significantly higher in patients with the anterior 
Fig. 1 Scatter plots of $\mathrm{ADC}_{1}$ and $\mathrm{AADI}(\mathbf{a}), \mathrm{ADC}_{1}$ and PADI (b). Spearman's coefficients were obtained, and regression lines were used for graphic purposes only
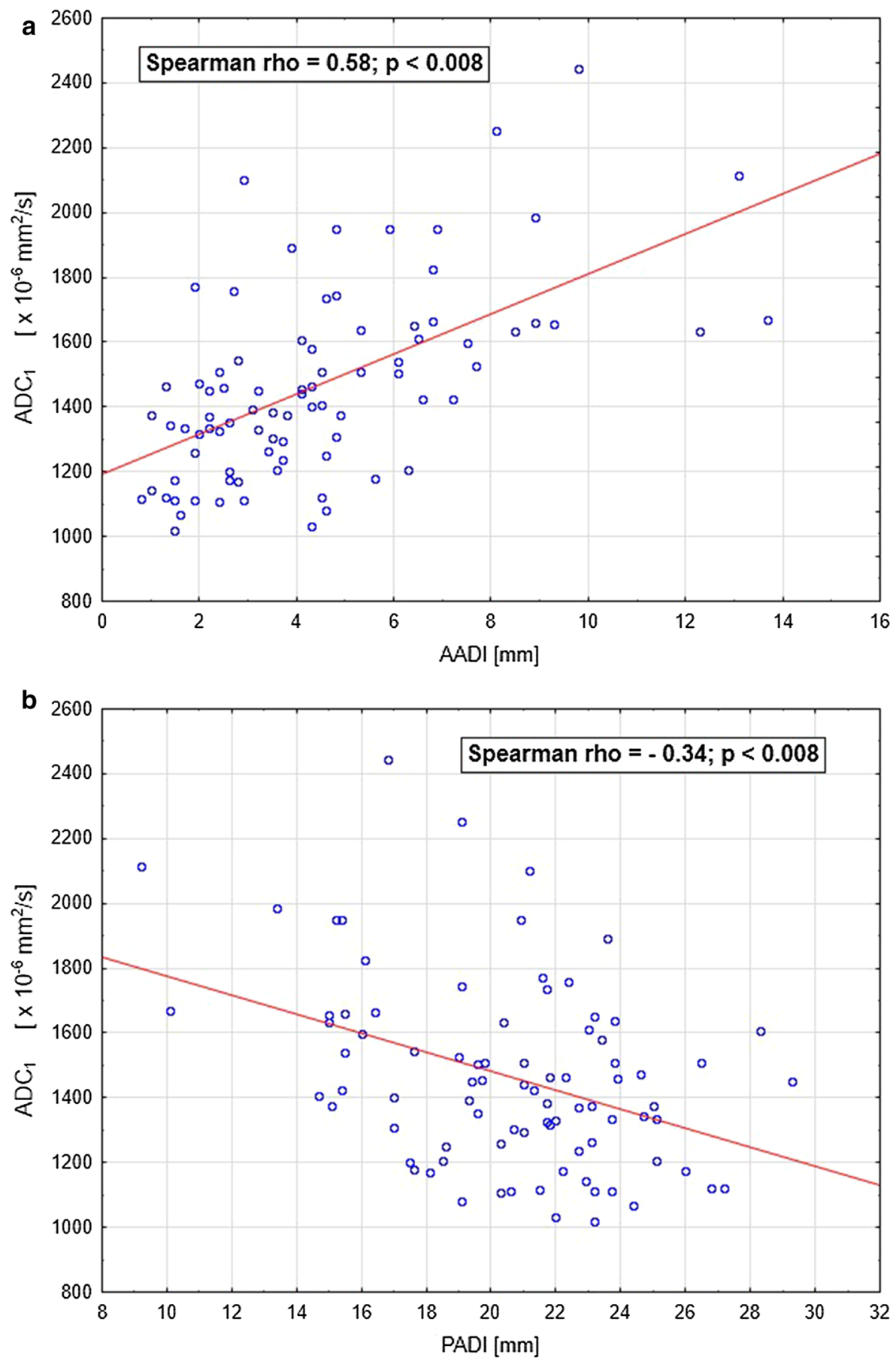

atlanto-axial instability than in patients without instability. These results are consistent with studies concerning diffusion parameters in patients with cervical degenerative myelopathy: The researchers described higher ADC values in patients with spinal canal stenosis in comparison with the control group [12-16], as well as higher ADC values in the area of spinal canal stenosis than in other parts of the spinal cord $[17,18]$. The high diffusion coefficient at the site of the spinal cord injury can be explained by mechanical cell damage caused by repeated pressure of the vertebrae (especially the dens) on the cord. This can cause damage to the neurolemma and myelinated fibers, disruption of cell membranes, which in turn allows for more free flow of water molecules (greater diffusion) in the intercellular space.

Many studies indicate high sensitivity of DWI and DTI parameters in detecting changes in a spinal cord caused by spinal canal stenosis, especially when comparing with conventional MRI. Demir et al. [19] demonstrated $61 \%$ 


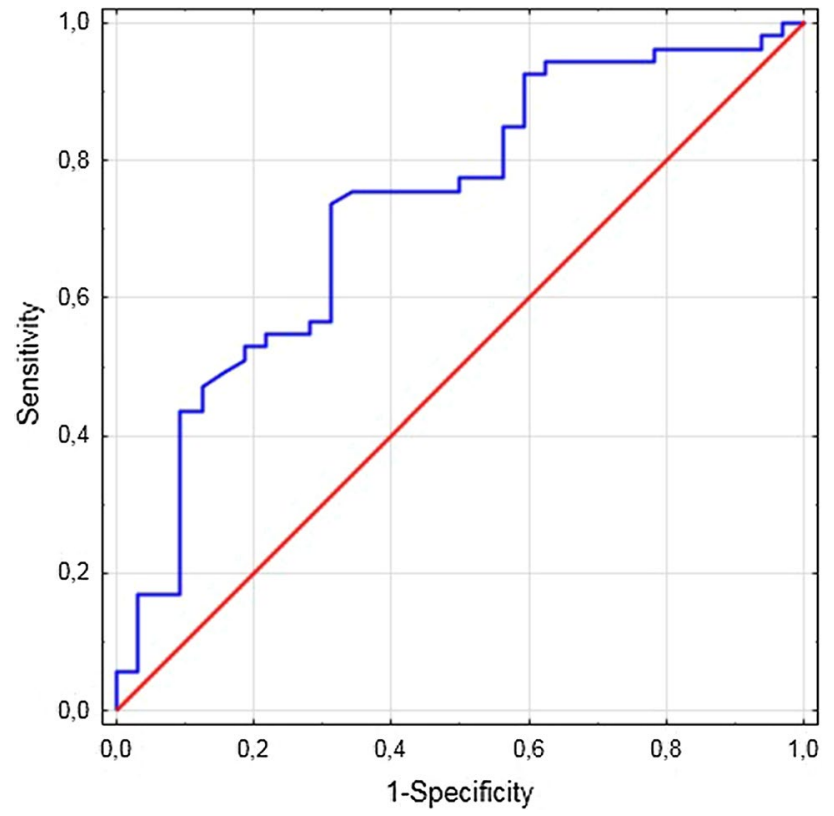

Fig. 2 ROC curve of $\mathrm{ADC}_{1}$

Table 2 Sensitivity, specificity and predictive values for $\mathrm{ADC}_{1}$ as a predictor of anterior AAS for different $\mathrm{ADC}_{1}$ cutoffs

\begin{tabular}{llccc}
\hline $\mathrm{ADC}_{1}$ cutoff & $\begin{array}{l}\text { Sensitiv- } \\
\text { ity }(\%)\end{array}$ & Specificity (\%) & PPV (\%) & NPV (\%) \\
\hline 1376 & 74 & 69 & 80 & 61 \\
1580 & 43 & 91 & 89 & 49 \\
1788 & 17 & 97 & 90 & 41 \\
2114 & 6 & 100 & 100 & 39 \\
\hline
\end{tabular}

sensitivity of T2-weighted images and $80 \%$ sensitivity of ADC maps in detecting myelopathy. Song et al. [10] observed hyperintense signal on T2-weighted images in $45 \%$ of patients with myelopathy, whereas changes on DTI images were observed in $74 \%$ of patients. The studies mentioned above described changes in the spinal cord caused by spinal stenosis at the level of $\mathrm{C} 2-\mathrm{C} 7$ vertebrae, whereas our study focused on pathology of the atlanto-axial region. In our study, despite the lack of changes in the signal intensity in atlanto-axial region on T1- and T2-weighted images, an increased diffusion coefficient was observed in patients with significant instability on radiographs. Thus, the ADC map may be more sensitive than T1- and T2-weighted signal intensities and may be able to detect pathology of the spinal cord earlier than conventional MRI.

In the presented work, it was possible to demonstrate a significant positive correlation between $\mathrm{ADC}_{1}$ and $\mathrm{AADI}$ value as well as to create a diagnostic test, in which $\mathrm{ADC}_{1}$ acts as a parameter allowing to classify the patient into the group with significant instability. With cutoff value of $1376 \times 10^{-6}\left(\mathrm{~mm}^{2} / \mathrm{s}\right)$ determined by Youden index, sensitivity amounted to $74 \%$ and specificity to $69 \%$. However, it is worth noting that with ADC value of $1788 \times 10^{-6} \mathrm{~mm}^{2} / \mathrm{s}$ the specificity increases up to $97 \%$. Therefore, it is very likely that in persons with the ADC values above $1800 \times 10^{-6}$ $\mathrm{mm}^{2} / \mathrm{s}$, a significant AAS occurs. The results of our study as well as the results of other researchers show that the ADC may be a helpful parameter to determine the degree of the spinal cord damage. Due to very large differences in the technical aspects of obtaining DWI images, it is currently not possible to define commonly applicable standards for diffusion parameters.

Many researchers have investigated the association of DWI and DTI parameters with neurological assessment. Kerkovsky et al. [12, 20] showed significant differences in diffusion features (both ADC and FA) between a group of patients with neurological symptoms of myelopathy and a group with asymptomatic myelopathy. In the group with symptomatic myelopathy, ADC was higher and FA was lower than in the latter group. Budzik et al. showed a significant positive correlation between FA values at the narrowed level of the spinal cord and the lower and upper limb functions, measured by the JOA (Japanese Orthopaedic Association) questionnaire. However, there was no similar relationship between ADC and any of the JOA questionnaire domains [21]. The present study did not confirm the relation between ADC and myelopathy symptoms, which is also consistent with the results of Lee et al. [22] who did not show any correlation between diffusion parameters (FA and ADC) and the severity of myelopathy.

In this study, no correlation between ADC values at six examined levels of the spinal cord and the age of patients has been demonstrated. One of the first studies on the imaging of diffusion in spinal cord diseases was the study by Mamata et al. [23], in which the authors indicate a weak positive correlation between the ADC and the age of patients $(r=0.242)$ and a weak negative correlation between the FA and the age $(r=-0.244)$. However, the study published more than 10 years later by Kerkovsky et al. [20] indicates the lack of correlation between the age and diffusion parameters. No correlation between the ADC values and the age of patients was found in the present study. Therefore, a statement that ADC values reflect the functional state of the spinal cord associated with the disease process and not with age seems to be justified.

The linear correlation between the AADI and $\mathrm{ADC}_{1}$ presented in this study is not ideal. The diagram shows points that are significantly far away from a simple regression. Therefore, it seems that the diffusion coefficient is not only a confirmation of the degree of radiographic instability, but it may also enrich the diagnostic process with the new insight. In the analyzed population, some 
patients were diagnosed with the low AADI values and high $\mathrm{ADC}_{1}$ values (see Fig. 1). It can be supposed that this relatively low instability, described by the AADI, is accompanied by the spinal cord damage.

Decision making in surgical treatment is currently carried out on well-recognized criteria based on functional $\mathrm{X}$-ray, MRI and the results of neurological examination. For now, diffusion parameters are not used in the patient's management, especially in the qualification for surgery. In the future, when the clinical role of ADC value will be better understood, maybe ADC will be an additional parameter in the qualification for surgical treatment. Probably high ADC values, regardless of normal AADI and PADI, may indicate spinal cord damage. Therefore, high ADC may encourage performing surgical intervention with intention to avoid myelopathy or more frequent than standard follow-up. However, this proposal of application of ADC has to be confirmed in the well-planned, prospective studies. For the time being, in the light of our results, DWI imaging should be incorporated into clinical practice, to deepen its potential application as well as monitor progression of damage.

\section{Conclusions}

The study demonstrated the usage of ADC values for identification of spinal cord compression due to the anterior atlanto-axial subluxation in RA patients, not yet detectable on standard cervical spine MRI. A positive correlation between the ADC values of the spinal cord at $\mathrm{C} 1$ level and the range of cervical spine instability at this level expressed with the AADI was found, which may indicate the applicability of the ADC maps in detecting structural changes of the spinal cord. For now, because diffusion parameters are not commonly used in clinical practice, it seems that increased ADC values-with small deviations in AADI and PADI - may entail more thorough monitoring of the patient. Introducing ADC maps into standard evaluation of spinal cord pathology requires improvements in the technical aspects of DWI with regard to artifacts, spatial resolution, as well as standardization of both protocols and postprocessing analysis, which will allow comparison of results between researchers.

\section{Compliance with ethical standards}

Conflict of interest The authors declare that they have no conflict of interest.
Open Access This article is distributed under the terms of the Creative Commons Attribution 4.0 International License (http://creativeco mmons.org/licenses/by/4.0/), which permits unrestricted use, distribution, and reproduction in any medium, provided you give appropriate credit to the original author(s) and the source, provide a link to the Creative Commons license, and indicate if changes were made.

\section{References}

1. Rooney BK, Silman AJ (1999) Epidemiology of the rheumatic diseases. Curr Opin Rheumatol 11(2):91-97

2. Helmick CG et al (2008) Estimates of the prevalence of arthritis and other rheumatic conditions in the United States. Part I. Arthritis Rheum 58(1):15-25

3. Myasoedova E et al (2010) Is the incidence of rheumatoid arthritis rising?: results from Olmsted County, Minnesota, 1955-2007. Arthritis Rheum 62(6):1576-1582

4. Bouchaud-Chabot A, Liote F (2002) Cervical spine involvement in rheumatoid arthritis. A review. Jt Bone Spine 69(2):141-154

5. Nguyen HV et al (2004) Rheumatoid arthritis of the cervical spine. Spine J 4(3):329-334

6. da Corte FC, Neves N (2014) Cervical spine instability in rheumatoid arthritis. Eur J Orthop Surg Traumatol 24(Suppl 1):S83-S91

7. Colebatch AN et al (2013) EULAR recommendations for the use of imaging of the joints in the clinical management of rheumatoid arthritis. Ann Rheum Dis 72(6):804-814

8. Neva MH et al (2006) High prevalence of asymptomatic cervical spine subluxation in patients with rheumatoid arthritis waiting for orthopaedic surgery. Ann Rheum Dis 65(7):884-888

9. Fujiwara $\mathrm{K}$ et al (2000) A long-term follow-up study of cervical lesions in rheumatoid arthritis. J Spinal Disord 13(6):519-526

10. Song $\mathrm{T}$ et al (2011) Diffusion tensor imaging in the cervical spinal cord. Eur Spine J 20(3):422-428

11. Nouri A et al (2017) The relationship between MRI signal intensity changes, clinical presentation, and surgical outcome in degenerative cervical myelopathy: analysis of a global cohort. Spine (Phila Pa 1976) 42(24):1851-1858

12. Kerkovsky M et al (2012) Magnetic resonance diffusion tensor imaging in patients with cervical spondylotic spinal cord compression: correlations between clinical and electrophysiological findings. Spine (Phila Pa 1976) 37(1):48-56

13. Chen $X$ et al (2016) Magnetic resonance diffusion tensor imaging of cervical spinal cord and lumbosacral enlargement in patients with cervical spondylotic myelopathy. J Magn Reson Imaging 43(6): 1484-1491

14. Rajasekaran S et al (2014) The assessment of neuronal status in normal and cervical spondylotic myelopathy using diffusion tensor imaging. Spine (Phila Pa 1976) 39(15):1183-1189

15. Banaszek A et al (2014) Usefulness of diffusion tensor MR imaging in the assessment of intramedullary changes of the cervical spinal cord in different stages of degenerative spine disease. Eur Spine J 23(7):1523-1530

16. Dong F et al (2018) A preliminary study of 3.0-T magnetic resonance diffusion tensor imaging in cervical spondylotic myelopathy. Eur Spine J 27(8):1839-1845

17. Toktas ZO et al (2016) Diffusion tensor imaging of cervical spinal cord: a quantitative diagnostic tool in cervical spondylotic myelopathy. J Craniovertebr Junction Spine 7(1):26-30

18. Nukala M et al (2019) Efficacy of diffusion tensor imaging in identification of degenerative cervical spondylotic myelopathy. Eur J Radiol Open 6:16-23 
19. Demir A et al (2003) Diffusion-weighted MR imaging with apparent diffusion coefficient and apparent diffusion tensor maps in cervical spondylotic myelopathy. Radiology 229(1):37-43

20. Kerkovsky M et al (2017) Spinal cord MR diffusion properties in patients with degenerative cervical cord compression. J Neuroimaging 27(1):149-157

21. Budzik JF et al (2011) Diffusion tensor imaging and fibre tracking in cervical spondylotic myelopathy. Eur Radiol 21(2):426-433

22. Lee JW et al (2011) Diffusion tensor imaging and fiber tractography in cervical compressive myelopathy: preliminary results. Skeletal Radiol 40(12):1543-1551
23. Mamata H, Jolesz FA, Maier SE (2005) Apparent diffusion coefficient and fractional anisotropy in spinal cord: age and cervical spondylosis-related changes. J Magn Reson Imaging 22(1):38-43

Publisher's Note Springer Nature remains neutral with regard to jurisdictional claims in published maps and institutional affiliations.

\section{Affiliations}

\section{Małgorzata Mańczak ${ }^{1}$ (D $\cdot$ Grzegorz Pracoń ${ }^{2} \cdot$ Iwona Sudoł-Szopińska ${ }^{2} \cdot$ Robert Gasik $^{3}$}

1 Department of Gerontology, Public Health and Didactics, National Institute of Geriatrics, Rheumatology and Rehabilitation, Spartańska 1, 02-637 Warsaw, Poland

2 Department of Radiology, National Institute of Geriatrics, Rheumatology and Rehabilitation, Spartańska 1, 02-637 Warsaw, Poland
3 Department of Neuroorthopaedics and Neurology, National Institute of Geriatrics, Rheumatology and Rehabilitation, Spartańska 1, 02-637 Warsaw, Poland 